\title{
La escuela infantil en el marco de la atención temprana: análisis de su organización escolar para la inclusión de la primera infancia
}

\author{
Álvarez Díaz, Katia \\ Universidad de Huelva, Huelva, España \\ Katia.alvarez@alu.uhu.es \\ González Falcón, Inmaculada \\ Universidad de Huelva, Huelva, Españal \\ Inmaculada.gonzalez@dedu.uhu.es
}

\section{Resumen}

La Escuela Infantil tiene un rol importante en el desarrollo físico, psíquico, y emocional del niño con necesidades a nivel biológico/psico-social inmerso en el marco de Atención Temprana. Esta importancia aún no es del todo reconocida por la sociedad, que sigue considerando este ciclo como un periodo evolutivo con relativo interés, pero lo valoran como un servicio que permite la conciliación de la vida familiar y laboral. Estos hechos provocaron que las guarderías, ahora llamadas escuelas infantiles, nacieran desde un enfoque asistencial sin intención educativa. Tras nuestra experiencia en este campo, se viene observando una tendencia a la intelectualización de este ciclo,asumiendo objetivos y metodologías propias. Cada época posee un modelo de escuela, y cada ámbito social reclama también cambios a la educación, de tal forma que su entorno debe ser transformado para renovar las posibilidades que se les ofrece a cada etapa. Tales cambios comienzan por asegurar espacios de mayor equidad, pertinencia e igualdad como reconocimiento a la inclusión educativa en la primera infancia. Desde este planteamiento educativo, la presente comunicación tiene por finalidad describir las características de una escuela infantil y su influencia en el desarrollo de la Atención Temprana, con el fin de analizar sus estructuras organizativas para atender a este colectivo en coordinación con otras entidades del ámbito sanitario y social. Para ello, se ha aplicado una metodología cualitativa, etnografía. Como conclusiones iniciales observamos cierta descoordinación interna y externa cuyas evidencias predicen la falta de comunicación entre los agentes que atienden a estos niños.

\section{Abstract}

The Nursery School an important role in the physical, psychic, and emotional development of the child with needs to level biológico/psico-social immersed in the frame of Early Attention. This importan cestillis not complete and admitted by the society, that he continues considering this cycle to be an evolutionary period with relative interest, but they value it as a service that allows the conciliation of the family and proyect life. These facts provoked that the day-care centers, now so called nursery school, were born from a welfare approach without inclusive education. After our experience in this field,one comes observing a trend to the intelectualitation of this cycle, assuming lenses and proper methodologies. Every epoch a model of school possesses, and every social are claims also changes to the education, in such a way that her environement must be transformed to renew the possibilities that one offers them to everystage. Such changes begin for assuring spaces of major equity, relevancy and equality as recognition of de inclusive education in the first infancy. From this educational exposition, the present communication has for purpose the characteristics of an nursery school and his influence in the development of the Early Attention, in order to analyze his organizational structures to attend to this group in coordination with other entities of the sanitary and social área. For it, a qualitative methodology, ethnography has been applied. As initial conclusions we observe certain internal and external uncoordinate whose evidences predict the lack of communication between the agents who attend to these children.

Palabras clave: Escuela Infantil, Atención Temprana, inclusión educativa, coordinación interdisciplinar. Keywords: Nursery School, Early Attention, inclusive education, coordination to interdiscipline. 


\section{INTRODUCCIÓN}

Los estudios dedicados a la atención temprana ( $A T$ en adelante) desde el ámbito educativo son cada vez más necesarios. Hoy día, ya se pueden ir observando en las Escuelas Infantiles la presencia de alumnado de 0-3 años con necesidades a nivel biológico/psico-social de distinta índole en su desarroIlo, o en altas posibilidades para que éstas aparezcan en un futuro más o menos próximo. No obstante, la intervención temprana en el primer ciclo de educación infantil recae generalmente en los CAITs ${ }^{1}$ mientras que, habitualmente, los apoyos que necesite el alumno escolarizado en segundo ciclo de esta etapa van a centrarse tanto en su propio centro escolar como en estas instituciones. Ante esta situación cabe preguntarse si existen y cuáles son los tipos de relaciones que mantiene la dirección de una El con otros agentes del ámbito educativo (Equipos Provincial de AT, familia, educadores), y desde el ámbito clínico (CAIT).

Justamente, todo ello depende de lo exitosa que sea la gestión de la AT en una El, es por tanto una preocupación para la mayoría de sus directores quienes se enfrentan día tras día a una etapa cada vez más diversa y heterogénea. Es por eso que los tres primeros años de escolaridad no obligatoria, tienen una especial importancia porque en el mismo se deben dar las garantías necesarias para que su papel de facilitador del desarrollo (físico, social e intelectual) y del descubrimiento en los discentes cumpla su cometido.

Existe todo un respaldo científico (García Sánchez, Sánchez López, Escorcia, Castellanos, 2012; Bolsanello, 2009; Arencibia, 2007; Pérez-López, 2010; Díaz Herrero, Martínez Fuentes, 2009 y Eloségui, Delinares y Casquero, 2003), que corrobora la importancia de llevar a cabo actuaciones de intervención educativa en este período para niños con distintas necesidades.

En este sentido, la AT desde e Ipunto de la intervención pedagógica se considera la posibilidad de abrir un mayor número de canales sensoriales que aporten al niño información sobre el mundo que le rodea y los recursos para que, a su vez, pueda interactuar y expresarse en el mismo.

Es por eso que a través de este estudio se defiende la idea de no considerar esta disciplina como un modelo individual de intervención alejada del aula, sino como aquella que incorpora múltiples recursos a la vida de los niños, como es el caso de la El. Institución escolar inmersa en los distintos que hacer es diarios de las clases y grupos, que potencian estas acciones en entornos naturales de desarrollo y aprendizaje y aprovecha, de esta forma, todas esas capacidades desde la posible necesidad (ya sea social, económica, o biológica) que manifieste el niño.

Una educación para la infancia, pero también por y con la infancia (González Falcón, Álvarez y Báez, 2014). Y es que en los últimos años, la educación y las necesidades de esta etapa están suscitando un gran interés a nivel internacional. Asimismo, la etapa de educación infantil (0 - 6años) es imprescindible por tener un marcado carácter preventivo y compensador para niños considerados de riesgo o dificultades en su desarrollo (Grande, 2011) en el que se les otorga un contexto de normalización para desarrollar sus potencialidades psicoevolutivas (Paniagua \& Palacios, 2005).

Justamente, el estudio tiene como premisa el derecho a una educación de calidad en la primera infancia, cuyo propósito recaiga en la diversidad como una característica positiva de la escuela y no como un pretexto de rechazo. Se considera, por tanto, al primer ciclo de educación infantil como uno de los periodos idóneos para compensar desigualdades no sólo de tipo económico y social, sino madurativas y evolutivas en los niños de 0 a 3 años, lo que hace que el empleo de los recursos de la AT y la EI sean dos marcos inmejorables para trabajar con juntamente como medios de prevención e intervención.

\section{LA EDUCACIÓN INFANTIL: CARACTERIZACIÓN DE LA ETAPA Y SU RELACIÓN CON LA ATENCIÓN TEMPRANA}

Desde una ventana muy particular: la Escuela Infantil como ámbito de experiencia, toca dirigir los ojos a la realidad concreta de la primera infancia, desvelar su foco de atención que será materia de este 
estudio, mirar lo que ella es y lo que se puede llegar a creer implícitamente en el trato que se le da en la familia, en las escuelas e incluso en otros colectivos sociales. Se considera que es una forma de explorar las representaciones conceptuales que de ella se ha ido ido elaborando a lo largo de los años; constituye una experiencia de reflexión constante que ya deporsí nos resulta valiosa para preguntarse qué se entiende y qué nos entiende, y para concebir una teoría, quizás no legitimada como la dispuesta en el contexto escolar.

La Educación Infantil concretamente, reviste una especial importancia en los primeros años de vida, en un sentido amplio, es aquella que se inicia desde el nacimiento del niño y que termina con el comienzo de la escolaridad obligatoria. Concretamente, se presenta como el tramo de enseñanza comprendido entre los 0 y 6 años del Sistema Educativo actual, dispuesta como una única etapa con características propias (Decreto 428/2008 de 29 de julio) y dividida en dos ciclos, el primero de 0 a 3 y el segundo de 3 a 6 años, teniendo ambos un carácter voluntario, no obligatorio.

Coincidiendo con la edad de intervención en AT, ésta presenta un marcado carácter preventivo y compensador para niños considerados de riesgo o con dificultades (GAT, 2000). En este sentido, la fundamentación psicopedagógica en la que se basa el currículo de esta etapa educativa constituye una plataforma privilegiada que justifica la intervención con los más pequeños tantoen el ámbito escolar, como en muchos casos, en el terapéutico (De Francisco Rodríguez, en Gútiez, 2005). Supone, por tanto, un contexto de normalización para atender a la diversidad ya que optimiza las potencialidades psicoevolitivas de todos los niños (Paniagua \& Palacios, 2005).

Es por tanto necesario conocer la relación existente entre la Educación Infantil y la AT a travésde las múltiples características que tienen en común (Grande, 2011; Pina, 2007).

a. La Educación Infantil y la AT presentan los mismos fines generales desde la optimización del desarrollo. Estos mismos objetivos son compartidos por los planteamientos de la AT, que, superando el modelo centrado en la discapacidad del niño, se orienta al desarrollo y máximo aprovechamiento de las posibilidades del mismo (GAT 2000).

b. Ambos sectores centran su trabajo en la atención planificada, intencional y sistemáticaen niños de entre 0-6 años. Este tipo de atención implica la interrelación de múltiples disciplinas (Gútiez 2005).

c. Se rigen por principios de universalidad, gratuidad, descentralización y sectorización (Belda, 2001). Además de compartir tales principios, la Educación infantil y la AT comparten otros tres principios fundamentales que las convierten en recursos absolutamente análogos: son los principios de intervención, globalización, individualización, integración e inclusión (GAT 2000).

d. Tanto la Educación Infantil y la AT comparten las funciones de prevención y compensación, educativa e integradora (Ruiz Veerman, 2005; Gómez Campillejo, 2009).

e. Principios didácticos y de intervención, en los que, de nuevo, tanto la educación infantil como la AT encuentran una total correspondencia:

- Principio de actividad: concibiendo al niño como protagonista de su aprendizaje.

- Principio del juego: actividad natural en estas edades y, constituyendo un importante motor del desarrollo, tanto en sus aspectos emocionales, como intelectuales y sociales.

- Principio de interés: implica que toda acción educativa ha de ser significativa, pero tanto para el niño como para el adulto.

Asimismo, el hecho de escolarizar al niño en edades tempranas favorece de manera positiva su desarrollo, siendo esta una de las medidas que se les aporta a la familia, de parte de los especialistas en AT quienes solicitan la escolarización en Primer Ciclo lo antes posible (Orteso, 2015).Ciertas investigaciones evidencian esta intervención, pues Pérez-López, Martínez-Fuentes, Díaz-Herrero y Brito (2012) llevaron a cabo un estudio sobre un grupo de niños que iniciaron su escolarización en el Primer Ciclo de Educación Infantil. Los resultados obtenidos informan que se observa en los sujetos un progreso mental y psicomotor favorable, entre el inicio y el final del curso escolar. En este sentido, Humphries y Keenan (2006) garantizan que la El es el medio principal para llevar acabo programas de prevención y argumentan que esta escolarización debe comenzar lo más pronto posible para maximizar su eficacia. 


\section{LA MICROPOLÍTICA DE LA ESCUELA INFANTIL: EN BUSCA DE UN LIDERAZGO INCLUSIVO}

Desde una postura analítica, en el proceso de simplificación para entenderla. El como organización educativa y asistencial encontramos que su realidad actual no es un todo homogéneo, es decir, la diversidad de aspectos que la configuran como: planteamientos educativos, estructurales y relacionales, sólo tienen sentido en la perspectiva de su totalidad personal y específica donde «los profesionales que en ella trabajan forman parte de una cultura que constituye un entramado de creencias, expectativas, normas y comportamientos» (Santos Guerra, 2002,p.23).

Actualmente, con la Escuela Infantil se pretende desarrollar en Andalucía un modelo educativo amplio y complementario al asistencial (Real Decreto 149/2009, de 12 abril) que se ha venido ofreciendo tanto para el ciclo 0-3 como para el 3-6 en las pasadas legislaturas. En este sentido la Escuela Infantil como institución educativa y asistencial debe ofrecer una doble respuesta: satisfacer las necesidades básicas del niño y de su familia en función a las necesidades de éstos, ocupándose de su alimentación, higiene, vigilancia y cuidados, junto al aprendizaje y desarrollo del niño en todas sus facetas, sin pretender acelerar el proceso, si no facilitar el progreso personal del mismo.

Para responder con éxito a tales demandas es necesario tomar un buen control funcional de la escuela; teniendo como referencia las argumentaciones de González González (2008), se muestran cinco dimensiones, utilizadas ambas como categorías de análisis, que debemos tener en cuenta en una institución escolar de estas características:

Tabla 1: Dimensiones para el estudio de la organización escolar.

\begin{tabular}{|c|c|}
\hline $\begin{array}{c}\text { Dimensión } \\
\text { Estructural }\end{array}$ & Elementos a partir de los cuales se ejecuta la acción institucional, es decir los órganos de gobierno. \\
\hline $\begin{array}{c}\text { Dimensión } \\
\text { relacional }\end{array}$ & Relaciones entre los individuos que la componen. \\
\hline $\begin{array}{c}\text { Dimensión } \\
\text { cultural }\end{array}$ & $\begin{array}{r}\text { Conjunto de significados, valores y, creencias compartidos por los miembros de la organización que le } \\
\text { otorgan una identidad propia. }\end{array}$ \\
\hline $\begin{array}{c}\text { Dimensión } \\
\text { procesal }\end{array}$ & Estrategias de actuación a través de las cuales la organización funciona. \\
\hline
\end{tabular}

Fuente: elaboración propia a partir de González González (2008).

Estos componentes de la Escuela Infantil se conjugan y entrelazan en una dinámica que da lugar a la propia particularidad de la organización (Antúnez, 2012) en la que se relacionan procesos con los matices propios de los sujetos que en ella conviven, por lo que cada Escuela Infantil, aún y cuando interactúen los mismos componentes será única y construirá su propia identidad personal. Dicha estructura organizativa tiene como objetivo plantear momentos de conexión entre todos estos elementos a partir de tareas de dinamización, coordinación de actividades en común, indispensables dentro de la organización de una escuela (Gómez Hurtado, 2012) la cual será la encargada de repartir funciones y competencias.

Resulta evidente que la composición de cada uno de estos elementos influirán en su modelo educativo y éste, en la estructura que llevará a su vez un modelo organizativo determinado en base a la gestión de la AT, ya que se requiere de una planificación exhaustiva, una intervención coordinada y una dedicación imperante. Es por eso que las tareas de coordinación entre los distintos agentes que conforma la Escuela Infantil y que viene siendo desarrolladas bajo la dirección para propiciar el buen desempeño de la AT, se consideran uno de los aspectos más influyentes para el desarrollo de un buen liderazgo inclusivo.

Por todo ello, se puede decir que no todos los directores poseen estilos de liderazgo que propicien políticas y culturas inclusivas. Cuando se habla de liderazgo inclusivo no sólo se refiere a la figura del director si no a la existencia de una dirección participativa y coordinada, que trabaja mejorando las condiciones de aprendizaje de todo su alumnado y, que es capaz de estimular y ofrecer procesos de reflexión y debate continuo entre todos los miembros que colaboran en la escuela. 
Para describir las distintas tareas que puede llegar a desempeñar la dirección en la gestión de la AT, el presente estudio se basará en el análisis elaborado por León (2001) sobre estudios y documentos relacionados con el tema de atención a la diversidad pueden aplicarse a esta investigación,donde se señala que para que se produzca el cumplimiento de las condiciones para el buen desempeño en su gestión la dirección debería:

- Promover la visión de «escuela como un todo».

- Ser el promotor de cambio y el encargado de controlar su desarrollo.

- Establecer relaciones de amistad y trabajo con toda la comunidad implicada en la escuela.

- Promover el desarrollo profesional del resto del equipo educativo y potenciar el trabajo cooperativo.

Leithwood et al (2004), tras la realización de un metanálisis identifica cuatro categorías que caracterizan las prácticas que llevan a un liderazgo exitoso:

- Establecer direcciones de coordinación.

- Rediseñar la organización espacio-temporal.

- Desarrollar a su equipo en términos de formación especializada.

— Gestionar la instrucción de enseñanza-aprendizaje en la escuela.

\subsection{Estrategias para gestionar la AT desde la EI}

Más allá de improvisar con urgencias, conviene establecer pautas y protocolos de actuación desde las direcciones en las Escuelas Infantiles que faciliten los procesos de toma de decisiones y favorezcan, asimismo, la cooperación institucional allá donde se produce el requerimiento. La dirección de una Escuela Infantil según Aranda y De Andrés (2004) debe partir de los siguientes principios:

- Diálogo entre las familias y las administraciones.

— Gratuidad e igualdad de oportunidades evitando riesgos y previniendo necesidades.

- Interdisciplinariedad (físico, psicológico y pedagógico).

- Coordinación de profesionales (CAIT, servicios sociales...).

- Buena sectorización que haga limitar los campos de actuación.

- Implicación del tutor de aula quien debe realizar una intervención didáctica teniendo en cuenta todos los factores del desarrollo infantil.

\section{OBJETIVOS Y METODOLOGÍA DE ESTUDIO}

La inquietud por describir y comprender la realidad de una Escuela Infantil como observatorio privilegiado en relación a la AT, hace concretar el perfil nuestro proceso metodológico. Las preguntas iniciales son: ¿Qué características de la Escuela Infantil y su contexto pueden incidir en la gestión de la AT? Para esclarecer los resultados sin pretensión generalizable, se ha optado por una metodología cualitativa, centrada concretamente en la etnografía (Latorre, Del Rincón y Arnal, 2003), cuyo interés es la descripción de una cultura concreta promovida desde la dirección de una Escuela Infantil situada en la capital onubense a través de un estudio de caso (Rodríguez, Gil y García, 1996). Los instrumentos empleados han sido: la observación, las entrevistas semiestructuradas y el análisis de documentos. Estas técnicas permiten hacer una triangulación de la información recogida y, de este modo, ofrecer datos lo más verídicos y reales posibles con el fin de no sesgar el análisis.

\section{DISCUSIÓN DE RESULTADOS}

La Escuela Infantil se mueve, a veces, en un terreno de incertidumbres que le obliga a superar ideas estereotipadas para buscar sentido a sus prácticas educativas. Caminar entre estas incertidumbres resulta más llevadero si se cuenta con un equipo capaz de debatir, dinamizar y abordar interrogantes, y de 
acordar criterios profesionales y éticos compartidos. Es por eso que toda organización escolar necesita de una toma de decisiones para su desarrollo, pero un paso previo es definir el lugar hacia el que se quiere ir, y en ese lugar sin duda, tendrá mucho que ver con el ideal de «una escuela con la infancia».

Teniendo en cuenta la primera dimensión, entorno, ésta se caracteriza por el escaso reconocimiento que a nivel social en este caso por parte de la familia y otro colectivo social (CAIT) aún se le da a la Escuela Infantil como entorno favorecedor para el tratamiento integral de estos niños.

En este centro educativo, en su concepto de escuela, representa un carácter asistencial y atencion al debido a que no la consideran como un objeto prioritario de intervención, si no más bien se concibe como un lugar para dar principalmente respuestas a las necesidades básicas de estos niños. Además, este hecho reafirma la escasez de casos que han sido atendidos en la propia escuela, principalmente tres desde su apertura hace ocho años; cuestiones comola sobre protección social ante las necesidades presentadas, la falta de realidad en cuanto a la unidad como etapa (0-6años), y el sobreesfuerzo económico al ser una entidad supeditada a una cuantía mensual, son algunas de las características socioculturales adscritas a la escuela que influyen directamente en el desarrollo de su propio reconocimiento funcional.

En cuanto al aspecto relacional, cabe destacar dos cuestiones: la escasa participación de la familias en la escuela y la inestabilidad de su plantilla; ambos condicionantes que generan gran controversia en la firmeza de su organización. Respecto a la familia ésta no considera al centro como un objetivo prioritario de intervención, sino más bien como un entorno de permanencia diferente al del hogar o los CAITs. Estos hechos reflejan el desconocimiento por parte de la familia en materia de AT, en el que ésta deja de ser participante activo de su intervención en la escuela para ser un mero transmisor de información.

Referente a la plantilla del centro. Otro de los aspectos es que es en este centro donde han tenido su primer contacto con niños con necesidades. La gran mayoría del equipo tanto educadoras como maestras, reconocen que no se sienten preparadas para atender a este alumnado, puesto que por un lado no han recibido formación en este ámbito, y por otro debido a la ausencia de casos encontrados en las escuelas.

Tomando como referencia estas últimas aclaraciones se atiende a la dimensión estructural de la escuela, en la que se evidencia una clara división de grupo «dirección-equipoeducativo» orientada hacia la verticalidad de un organigrama claramente jerárquico, y donde el intercambio de información suele producirse de manera unidireccional, lo que provoca una falta de autonomía e iniciativa por parte del equipo educativo en la escuela cuanto a sus prácticasinclusivas.

La dimensión cultural promovida por la comunidad educativa acerca de la AT, también refleja una acción particular a expensa de colaborar en un equipo interdisciplinar promovido desde los diferentes entornos a lo sque acude el niño.Desde esta perspectiva Gútiez \& Rúiz Veerman (2012) abogan por un cambio del modelo educativo en la escuela hacia un modelo educativo compartido alcanzando el máximo grado de interacción social entre los distintos entornos en los que es atendido.

Desde la dimensión procesual, cabe destacar la inexistencia de un plan integral e interdisciplinar de actuación en la El, reflejando la clara individualización y descoordinación entre ambas entidades inmersas en el sistema de AT. En este caso, la dirección del centro presenta como medio para favorecer las actuaciones con la alumna en AT, el programa de estimulación motriz CEMEDETE a través del cual evidencia una propuesta globalizada de intervención a nivel de escuela que no está supeditada al programa de intervención elaborado desde el CAIT .

\section{CONCLUSIONES}

Tras verificar los resultados obtenidos se puede decir que los cuatro pilares principales para que se desarrolle con éxito la intervención temprana en la El pasa por atender en primer lugar a la organización escolar como una institución capaz de asistir, educar e intervenir; en segundo lugar por conocer la existencia de recursos (humanos y materiales) que éstos tengan a su serviciopara el apoyo y seguimiento de la intervención y, en tercer lugar, por atender a una formación más específica de su equipo educativo en materia de intervención psicoterapeuta y, por último, atender a la coordinación entre los diversos ámbitos deactuación. 
Desde este estudio de caso, se ha evidenciado que la Escuela Infantil presenta la necesidad de un cambio en su gestión que pase por concebir la escuela como una institución educativa de apoyo a la intervención e inclusión compartida, cuyo objetivo y de acuerdo con León (2001) sea proporcionar una respuesta organizada a las necesidades sociales de educación y AT, y no como un mero ejercicio aislado que aboga por la intervención a nivel de centro.

Asimismo, las propuestas actuales de intervención en AT plantean la necesidad de potenciar una cultura escolar propulsora y comprometida en la búsqueda hacia la inclusión, donde el propio equipo educativo, la familia y CAIT sean un solo eslabón de este sistema de atención infantil temprana. De esta forma, se avanza hacia la dirección escolar como un factor clave para mejorar la escuela, pues de acuerdo con Corbellas (2016) se está en la búsqueda de una dirección competente viendo que en todos los modelos de mejora aparece el perfil de dirección como un elemento imprescindible y sus intervenciones como un elemento diferenciador de la capacidadde cambiar la escuela. En dicho cambio, la familia desempeña un papel importante, para favorecer y potenciar sus intervenciones en la escuela colaborando en el diseño de su plan integral, en la búsqueda de recursos y aportando la información necesaria a la escuela.

Para ello, se considera primordial la coordinación de ambos centros, puesto que por un lado, servirá para dar a conocer al niño y sus características, pero también para favorecer la continuidad y enriquecer los objetivos de intervención planteados de la AT. Por todo ello, desde los principios ya comentados a lo largo del análisis, es fácil entender que no sólo se ha de potenciar esa coordinación, sino que también se ha de realizar esfuerzos necesarios para evaluar y seguir esa coordinación.

A este respecto se expone algunas de las investigaciones que actualmente están inmersas en los procesos de éxito para la búsqueda de la inclusión educativa en la escuela; Castellanos, Mendieta, Rico y Sánchez (2015), representan en sus estudios sobre buenas prácticas a través de un Programa de Coordinación con Escuelas Infantiles la filosofía de actuación interdisciplinar, cuyo objetivo es mantener abiertos canales ágiles de comunicación entre sus profesionales, los cuales faciliten la inclusión del niño y permitan contribuir a la tarea de fomentar y potenciar estos entornos competentes; los objetivos propuestos se centran en; liberar de atención directa a profesionales para el desarrollo de actuaciones en la El; fomentar y desarrollar reuniones de coordinación interprofesionales; concienciar a las familias de la necesidad de asistencia del niño a la El como complemento de su programa individual de intervención; Sensibilizar al personal docente y crear una actitud positiva, abierta y colaboradora hacia la coordinación; incluir en el Programa Individual de AT objetivos a trabajar en la El; informar y apoyar a los profesionales de la El en el programa de AT del niño y ajustar los objetivos a trabajar en su entorno según las posibilidades decada El; proponer y ofrecer apoyo y asesoramiento al personal de la El, asistiendo con asiduidad al aula para observar al niño y servir como modelo de actuación; atender las demandas y dudas que desde las EI se realicen al CDIAT y evaluar anualmente el Programa reflejando los resultados en la memoria correspondiente e incluyendo propuestas de mejora.

\section{REFERENCIAS BIBLIOGRÁFICAS}

Antúnez, S. (2012). Una brújula para la dirección escolar. Orientaciones para la mejora. México: SM Ediciones.

Arencibia, E. (2007). Atención Temprana desde la perspectiva sistemática. Análisis y estudio de un caso Y. Revista de Atención Temprana, 2 (3), 105-111.

Belda, J. y Casbas, M. (2013). Saber lo que no hay que hacer en Atención Temprana. Desenvolupament Infantil I Atenció Precoç. 34,1-8.

Bolsanello, M.A. (2009). Prevención desde la Escuela Infantil: desafíos en la realidad brasileña. Revista Interuniversitaria de Formación del Profesorado. 65 (4), 73-82.

Corbella,M.R.(2016).Liderazgo y responsabilidad educativa: el necesario liderazgo de directores y profesores en la educación. Revista Fuentes, (14), 85-104.

Díaz Herrero, A. y Martínez Fuentes, M.T. (2009). Prevención y promoción del desarrollo infantil: una experiencia en las Escuelas Infantiles. Revista Interuniversitaria de Formación del profesorado. 65 (6), 57-72. 
Élosegui, E. De Linares, C., y Casquero, D. (2003). Estudio sobre la necesidad de formación en Atención Temprana. Revista de Atención Temprana. 6 (2), 6-10.

García, F.A., Sánchez, M.C., Escorcia, C.T., y Castellanos P. (2012). Valoración de la Coordinación entre Atención Temprana y Educación Infantil por Educadores de Escuelas Infantiles. Revista Edetania, 41 (2), 145-161.

Gómez-Hurtado,l.(2012).Dirección escolar y atención a la diversidad: rutas para el desarrollo de una escuela para todos. (Tesis Inédita de doctorado). Universidad de Huelva:Huelva.

González González, M.T. (2008). Diversidad e inclusión educativa. Algunas reflexiones sobre liderazgo en el centro escolar. Revista Electrónica sobre Calidad, Eficacia y Cambio en Educación, 6, (2), 82-99.

González, I., Álvarez, K., y Báez, C. (2014). El juego en la infancia. Una experiencia innovadora en el Grado de Educación Infantil. En S. Jiménez, A. Pantoja, J.J. Leiva, E. Moreno yJ.D. Gutiérrez (Coord.), Actas de las Comunicaciones del Congreso Internacional Infancia en contextos de riesgo (3530-3543): Málaga: Universidad deMálaga.

Grande, P. (2011). Estudio de la coordinación interinstitucional e interdisciplinar en Atención Temprana en la comunidad de Madrid. La experiencia de un programa marco de coordinación de Getafe. Tesis Inédita de la Universidad Complutense, Madrid.

Grupo de AtenciónTemprana (2005). Recomendaciones técnicas para el desarrollo de la Atención Temprana. Madrid: Real Patronato sobre Discapacidad.

Gútiez, P. (2005). Atención Temprana. Prevención, detección e intervención en el desarrollo (0- 6 años) y sus alteraciones. Madrid: Ed. Complutense.

Gútiez, P. y Ruíz, E. (2012). Orígenes y evolución de la Atención Temprana. Una perspectiva histórica de la génesis de la Atención Temprana en nuestro país. Agentes, contextos y procesos. Revista Psicología Evolutiva, 18(2),12-22.

Humphries, M. L.y Keenan, K.E. (2006). Theoretical, developmental and cultural orientations of school-based prevention programs for preschoolers. Clinical child and family psychology review. 9, 2, 135-48.

Leithwood, K. (2004). El liderazgo con éxito. El liderazgo educacional transformador en unmundo de políticas transnacionales. En Villa, A. (Coord.). Dirección para la incorporación: Apertura de los centros a la sociedad del Conocimiento. Bilbao: Universidad deDeusto.

León Guerrero, M. J. (2001). La dirección de las instituciones educativas y la atención a la diversidad. En De Vicente Rodríguez, P.S. Viaje al centro de la dirección de instituciones educativas. (399-417). Bilbao: Ediciones Mensajero.

Orteso, F. J. (2015).Atención temprana desde una perspectiva educativa: «análisis de la población atendida por un equipo de orientación educativa y psicopedagógica (de atención temprana)». Tesis inédita de doctorado. Universidad De Murcia. Facultad de Psicología. Murcia.

Paniagua, G. y Palacios, J. (2005). Educación Infantil:r espuesta educativa a la diversidad. Madrid: Alianza.

Pérez-López, J. y Brito De La Nuez, A. (Coords.) (2010). Manual de Atención Temprana. Madrid: Pirámide.

Pina, J. (2007). Análisis de un modelo de seguimiento en atención temprana. (Tesis inédita de doctorado). Universidad Complutense, Madrid.

Ruiz Veerman, P. (2005). La formación de los profesionales en deficiencias y discapacidad de la primera infancia. (Tesis inédita de doctorado). Universidad Complutense, Madrid.

Santos Guerra, M. A. (2002). Organizar la diversidad. Cuadernos de pedagogía, 311,(s), 76-80. 